\title{
CYP2C19 genotype-guided antiplatelet therapy: promises and pitfalls
}

\author{
Moataz Ellithi ${ }^{1}$ D , Jordan Baye ${ }^{1}$ \& Russell A Wilke*,1 \\ ${ }^{1}$ Department of Internal Medicine, University of South Dakota Sanford School of Medicine, Sioux Falls, South Dakota, SC \\ 57105, USA \\ *Author for correspondence: Moataz.Ellithi@USD.edu
}

Pharmacogenetic variants can alter the mechanism of action (pharmacodynamic gene variants) or kinetic processes such as absorption, distribution, metabolism and elimination (pharmacokinetic gene variants). Many initial successes in precision medicine occurred in the context of genes encoding the cytochromes P450 (CYP enzymes). CYP2C19 activates the antiplatelet drug clopidogrel, and polymorphisms in the CYP2C19 gene are known to alter the outcome for patients taking clopidogrel in the context of cardiovascular disease. CYP2C19 loss-of-function alleles are specifically associated with increased risk for coronary stent thrombosis and major adverse cardiovascular events in patients taking clopidogrel following percutaneous coronary intervention. We explore successes and challenges encountered as the clinical and scientific communities advance CYP2C19 genotyping in the context of routine patient care.

First draft submitted: 30 March 2020; Accepted for publication: 29 April 2020; Published online: 29 July 2020

Keywords: clopidogrel • CYP2C19 • drug-metabolizing enzyme • pharmacogenomics • pharmacokinetics • pleiotropy $\bullet$ polymorphism $\bullet$ Prasugrel $\bullet$ precision medicine $\bullet$ Ticagrelor

Cardiovascular diseases (CVD), which include coronary artery disease (CAD), peripheral arterial disease (PAD) and cerebrovascular disease, remain the leading cause of death worldwide [1]. CVD represents a spectrum of diseases that share similar pathophysiology, and platelets play a pivotal role in this pathophysiology by regulating the development of chronic atherosclerotic plaques and triggering molecular cascades that lead to the progression of arterial thrombosis in response to atherosclerotic plaque rupture [2]. Thus, antiplatelet therapy is considered a cornerstone in the treatment and prevention of CVD. A list of common antiplatelet drugs used in CVD treatment and prevention is outlined in Table 1.

Aspirin was one of the first antiplatelet agents to show benefit in patients with CVD. Through irreversible inhibition of COX enzymes, particularly COX-1, aspirin blocks the production of thromboxane A2, which is a vasoconstrictor and a stimulant for platelet aggregation. Aspirin thereby reduces thrombus formation and CVD events. While the use of aspirin as monotherapy in the context of primary prevention remains a topic of ongoing debate [3-5], aspirin has been shown to have great efficacy in secondary prevention of cardiovascular diseases, especially when combined with other antiplatelet medications [6]. In patients with known CVD, aspirin reduces the risk of nonfatal myocardial infarction and stroke by 26 and $25 \%$, respectively, and it further reduces all-cause mortality by $13 \%[7]$.

Adenosine diphosphate (ADP) stimulates platelet aggregation in vivo, and P2Y12 antagonists (purinergic receptor P2Y, G-protein-coupled 12 adenosine diphosphate receptor antagonists) are potent inhibitors of platelet aggregation. Ticlopidine was among the first clinically available P2Y12 antagonists, but its use was associated with hematologic adverse events such as neutropenia and thrombotic thrombocytopenic purpura [8]. Newer, safer P2Y12 antagonists eventually emerged in routine clinical practice, including clopidogrel and prasugrel. The clinical benefit of adding clopidogrel to aspirin therapy in acute CAD has been demonstrated in numerous large, multicenter, randomized controlled trials [9-11]; however, the combination has failed to show superior clinical efficacy, compared with aspirin alone, within stable CAD.

Acute coronary syndromes are now often treated by percutaneous coronary intervention (PCI), and prescription of dual antiplatelet therapy (DAPT) with a P2Y12 antagonists such as clopidogrel plus aspirin has become 
Table 1. List of antiplatelet medications along with their mechanism and forms of metabolism.

\begin{tabular}{|c|c|c|c|}
\hline Drug & Route & Mechanism of action & Metabolism \\
\hline Aspirin & Oral & COX inhibitor, nonselective & Hydrolysis by tissue esterases \\
\hline Cilostazol (Pletal) & Oral & Phosphodiesterase III inhibitor & Oxidation by CYP3A4 (major) and CYP2C19 (minor) \\
\hline $\begin{array}{l}\text { Dipyridamole (Persantine and } \\
\text { Aggrenox) }\end{array}$ & Oral & $\begin{array}{l}\text { Adenosine uptake inhibitor; phosphodiesterase } \\
\text { inhibitor }\end{array}$ & Glucuronidation \\
\hline Ticlopidine (Ticlid) & Oral & $\mathrm{P}_{2} \mathrm{Y}_{12}$ inhibitor (irreversible) & $N$-dealkylation and oxidation \\
\hline Clopidogrel (Plavix) & Oral & $\mathrm{P} 2 \mathrm{Y}_{12}$ inhibitor (irreversible) & $\begin{array}{l}\text { Oxidation by CYP2C19 (major) }{ }^{\dagger}, \text { CYP3A (minor), } \\
\text { CYP2B6 (minor) }\end{array}$ \\
\hline Prasugrel (Effient) & Oral & $\mathrm{P}^{2} \mathrm{Y}_{12}$ inhibitor (irreversible) & $\begin{array}{l}\text { Oxidation by CYP3A4 (major) CYP2B6 (major), } \\
\text { CYP2C9 (minor), CYP2C19 (minor) }\end{array}$ \\
\hline Ticagrelor (Brillinta) & Oral & $\mathrm{P}_{2} \mathrm{Y}_{12}$ inhibitor (reversible) & Oxidation by CYP3A4 \\
\hline Cangrelor (Kengreal) & IV & $\mathrm{P}_{2} \mathrm{Y}_{12}$ inhibitor (reversible) & Dephosphorylation \\
\hline Eptifibatide (Integrilin) & IV & Glycoprotein Ilb/IIla inhibitor (reversible) & None \\
\hline Abciximab (Reopro) & IV & Glycoprotein Ilb/Illa inhibitor (irreversible) & Proteolytic cleavage \\
\hline Tirofiban (Aggrastat) & IV & Glycoprotein Ilb/IIla inhibitor (reversible) & None \\
\hline
\end{tabular}

the standard of care to maintain stent patency and prevent recurrent coronary events [10,12]. In large populations, however, some patients using DAPT fail to achieve the desired therapeutic outcome from clopidogrel. Newer P2Y12 antagonists such as prasugrel and ticagrelor may deliver improved efficacy in specific patient subsets. In the Trial to Assess Improvement in Therapeutic Outcomes by optimizing platelet Inhibition with Prasugrel Thrombolysis In Myocardial Infarction 38 (TRITON-TIMI 38), patients receiving prasugrel after PCI for an acute coronary syndrome had a lower incidence of major adverse cardiovascular events (MACE) compared with patients receiving clopidogrel (9.9 vs 12.1\%) [13,14]. In the Platelet Inhibition and Patient Outcomes (PLATO) trial, patients receiving ticagrelor after PCI for an acute coronary syndrome also had a lower incidence of MACE compared with patients receiving clopidogrel [15]. Following these landmark trials, both prasugrel and ticagrelor have recently received a class I recommendation for their use in patients who have acute coronary syndromes with or without ST-segment elevation $[16,17]$. The use of clopidogrel is therefore increasingly being limited to situations where prasugrel and ticagrelor are not well tolerated.

The choice of which P2Y12 antagonist to select within a DAPT regimen after PCI is complex. There is wide variability in cost between clopidogrel, prasugrel and ticagrelor, and each patient's risk for developing toxicity depends upon their prior individual medical history. For example, in some patients, prasugrel has been associated with a higher risk of bleeding than clopidogrel, and prasugrel is therefore contraindicated in patients with a prior history of stroke or transient cerebral ischemia [18,19]. All of these factors - cost, variability in efficacy and variability in toxicity - influence patient adherence. At present, the choice of P2Y12 antagonist is made based upon burden of disease, bleeding risk and clinical factors such as concomitant medication (drug-drug interaction). A key unanswered question has been whether the field of genetics (drug-gene interaction) should be leveraged to further guide clinicians with drug selection.

\section{CYP2C19}

In general, P2Y12 antagonists undergo considerable Phase I oxidation by cytochromes P450 (Table 1). Clopidogrel in particular is primarily metabolized by CYP2C19 [20]. Because the CYP2C19 gene is highly polymorphic, and because clopidogrel is a prodrug requiring bioactivation by this enzyme in vivo, the association between CYP2C19 gene variants and clopidogrel efficacy appears to be clinically actionable. Patients with 'loss of function' CYP2C19 alleles have lower levels of active clopidogrel metabolites, and these patients have reduced efficacy (less platelet inhibition) when compared with patients with normal alleles [21-26]. Further, loss of function CYP2C19 alleles are common in patients of all ancestry (Table 2). Approximately $28 \%$ of Europeans have at least one loss of function allele, $42 \%$ of Africans have at least one loss of function allele and $59 \%$ of Asians have at least one loss of function allele [26-28].

The CYP2C19 gene maps to the long arm of chromosome 10 (10q24) and it encodes a 490-amino-acid protein predominantly expressed in the liver and, to a lesser extent, in the small intestine. The most common nonfunctional 
Table 2. Frequency of CYP2C19 enzyme phenotype by major continental race.

\begin{tabular}{|llll|}
\hline CYP2C19 phenotype & European ancestry & African ancestry (sub-Saharan) & Asian ancestry \\
\hline PM & $2 \%$ & $6 \%$ & $13 \%$ \\
\hline IM & $26 \%$ & $36 \%$ & $46 \%$ \\
\hline RM & $40 \%$ & $30 \%$ & $38 \%$ \\
\hline UM & $27 \%$ & $19 \%$ & $3 \%$ \\
\hline Uncertain & $5 \%$ & $3 \%$ & $0 \%$ \\
\hline
\end{tabular}

Frequencies summarized from populations in the Clinical Pharmacogenetics Implementation Consortium. [27].

IM: Intermediate metabolizer; PM: Poor metabolizer; RM: Rapid metabolizer; UM: Ultrarapid metabolizer.

allele, CYP2C19*2, has an estimated minor allele frequency (MAF) of approximately $15 \%$ in Europeans and Africans, and approximately $30 \%$ in Asians [29]. The CYP2C19*2 allele $(681 \mathrm{G}>\mathrm{A})$ results in a splicing event that causes premature termination of the protein and leads to a malfunctioning enzyme [30]. Another nonfunctional allele, $C Y P 2 C 19^{*} 3(636 \mathrm{G}>\mathrm{A})$, is also relatively common in Asian populations (up to $10 \%$ are carriers) [27]. Other CYP2C19 gene variants that lead to loss of function are relatively rare.

It is also important to note that CYP2C19 gene variants do not account for all of the variability in clopidogrel response. Transcriptional activation of this enzyme is mediated by drug-responsive nuclear receptors such as CAR (NR1I3), PXR (NR1I2) and GR $\alpha$ (NR3C1), suggesting regulation by endogenous hormones and metabolic interference by drugs such as rifampin, ritonavir and dexamethasone [31,32]. Further, pharmacodynamic gene variants in the purinergic receptor and/or in the G-proteins that transduce its molecular signal may also alter outcome for clopidogrel [33]. Combinatorial models including pharmacodynamic genes (e.g., P2RY12), and pharmacokinetic genes beyond CYP2C19 (e.g., CYP2B6 and CES1), may therefore provide even greater power to improve the outcome for antiplatelet therapy $[34,35]$.

\section{Promises}

The Clinical Pharmacogenomics Implementation Consortium (CPIC) regularly publishes guidelines for the use of pharmacodynamic and pharmacokinetic drug-gene relationships [36,37]. To date, CPIC has produced 24 guidelines pertaining to 62 medications and 20 pharmacogenes (https://cpicpgx.org/guidelines/). These guidelines provide specific guidance for clinical situations where pharmacogenetic test results are already available; they do not, however, determine when a patient should be genotyped. The decision to genotype should be made by individual patients and clinicians within the context of routine care. Factors influencing the clinical actionability of a druggene relationship vary by drug, and they typically include therapeutic index as well as the potential clinical severity of any adverse drug reactions [38]. Examples of drugs that meet these criteria include psychotropic agents, immune modulators, chemotherapeutics and drugs used to modulate hemostasis. Antiplatelet agents specifically have shown great promise in terms of reducing adverse events when a gene-based prescribing approach is applied.

In general, clinician response to pharmacogenetic information will vary depending upon the drug. In some situations, it may be best to adjust the dose based upon genotype - increasing the dose to avoid therapeutic failure or decreasing the dose to avoid toxicity. In other situations, it may be best to change the drug altogether - selecting an alternate agent within the class or moving to a different class of drugs. CPIC guidelines explore each of these approaches, and the specific guidance provided in each guideline varies drug by drug and gene by gene. Essentially, three simple paradigms are emerging. When a patient is identified to have an actionable pharmacogene variant, providers can either change drug, change dose or make no initial change while monitoring more closely (at increased frequency). Each of these paradigms is explored below, specifically for CYP2C19 genotype and antiplatelet therapy.

\section{Change the drug}

Over the past decade, CYP2C19 genotype has unequivocally been associated with cardiovascular event rate in patients using clopidogrel after percutaneous coronary intervention. Patients using clopidogrel in TRITON-TIMI 38 had a threefold increased risk for coronary stent thrombosis if they were carriers of a CYP2C19 loss of function allele [24]. These same patients had 50\% increase in risk for a combined MACE end point (fatal and non-fatal MI, stroke and cardiovascular death) [24]. This drug-gene relationship therefore appears to be actionable in heterozygotes 
(where even one loss of function allele attenuates bioactivation of clopidogrel in vivo, placing that patient at threefold increased risk of therapeutic failure).

Subsequent data have indicated that clinicians may be able to avoid therapeutic failure in the context of patients with CYP2C19 loss of function alleles if carefully selected patients are switched from clopidogrel to prasugrel or ticagrelor. In a genetic substudy of PLATO, ticagrelor reduced MACE events more than clopidogrel in IMs (intermediate metabolizers) and PMs (poor metabolizers), an effect that was larger in IMs and PMs than in patients without a loss of function allele (hazard ratio [HR] of $0.77 \mathrm{vs} 0.86$, respectively) [15]. More recently, a prospective study of 628 PCI patients in China demonstrated that genotype-guided therapy carried a $55 \%$ lower risk of MACE at 12 months (with no difference in bleeding outcomes) when compared with conventional therapy with clopidogrel [39].

Definitive data are now emerging in support of the claim that CYP2C19 genotype can be leveraged to reduce adverse cardiovascular events by switching PCI patients with a loss of function CYP2C19 allele from clopidogrel to prasugrel or ticagrelor if they do not have contraindications to these alternate P2Y12 antagonists. The Implementing Genomics in Practice (IGNITE) research network recently replicated findings from TIMI-38 in a practice-based cohort (50\% increase in risk for MACE on clopidogrel, in PCI patients with one loss of function CYP2C19 allele), and they extended those findings to demonstrate that changing the drug (from clopidogrel to prasugrel or ticagrelor) normalized the risk for recurrent MACE events in these patients [24,40].

This strategy of changing drug in select patients (without contraindication) is being widely adopted by organizations such as the Royal Dutch Pharmacists Association Pharmacogenetics Working Group (DPWG) and the US FDA. FDA recently announced a warning on the clopidogrel label recommending consideration of alternative therapies in patients who are CYP2C19 poor metabolizers as they may not receive the full benefits of the drug. CPIC therefore recommends changing the drug - from clopidogrel to prasugrel or ticagrelor - when most patients with a loss of function CYP2C19 allele require therapy with a P2Y12 antagonist [30]. In situations where patients with a loss of function CYP2C19 allele have a contraindication to prasugrel (e.g., prior stroke), or side effects with ticagrelor (e.g., drug-induced dyspnea), in vitro data suggest that changing the dose of clopidogrel may be helpful; however, this has not yet been shown to reduce cardiovascular events. To guide clinicians through the implementation of these recommendations within the context of a busy clinical practice day, automated decision support is now being deployed in many commercial electronic medical records [41].

\section{Change the dose}

As noted above, there are some situations where changing the drug may not be feasible. Although ticagrelor and prasugrel have been shown to reduce thrombotic events more than clopidogrel in patients with an acute coronary syndrome [15,42], ticagrelor and prasugrel are also associated with higher bleeding risk [15,43]. Further, cost concerns (especially for agents still under exclusivity) may prohibit their use for prolonged therapy. Thus, some patients prefer to stay on clopidogrel despite the presence of a CYP2C19 gene variant, raising the issue of whether increased doses of clopidogrel ( $150 \mathrm{mg}$ or $225 \mathrm{mg}$ daily) may be able to overcome the partial decrease in CYP2C19 enzyme activity seen in patients with only one loss of function allele. If successful, an increase in parent drug (doubling or tripling the clopidogrel dose in heterozygotes) could conceivably overcome the enzyme deficit and provide sufficient antiplatelet effect to normalize the risk without incurring the added cost of ticagrelor or the bleeding risk of prasugrel.

Studies done in vitro using platelets harvested from patients taking different doses of clopidogrel in the Escalating Clopidogrel by Involving a Genetic Strategy - Thrombolysis in Myocardial Infarction 56 (ELEVATE-TIMI 56) trial have leveraged a platelet activation (VASP) assay to demonstrate that a CYP2C19 enzyme deficit can be partly overcome by dose escalation [44,45]. Mega et al. observed a dose-dependent increase in platelet inhibition in both heterozygotes and homozygotes suggesting that this approach may be effective [45]. However, no clinical outcome data have shown that this approach (i.e., changing dose rather than changing drugs) actually reduces the frequency of MACE events. Thus, since data have only shown lab-based efficacy by dose escalation, rather than clinical efficacy (event reduction) by dose escalation, this approach has largely been reserved for situations where either the patient cannot afford the cost of switching drugs, or the patient has other clinical comorbidities that preclude the use of an alternate P2Y12 antagonist, such as age older than 75 years with prasugrel or the development of dyspnea as an adverse drug reaction with ticagrelor $[46,47]$. 


\section{Make no change (monitor closely)}

Another approach that is often considered with pharmacogenetic data is opting not to change medication, but instead to monitor patients more closely in an attempt to avoid potential adverse outcomes. While such an approach may be appropriate in the context of some drugs metabolized by CYP2C19, it is not advisable for the gene-based prescribing of antiplatelet agents. For example, CYP2C19 oxidizes proton-pump inhibitors (PPIs), tricyclic antidepressants (TCAs) and selective serotonin reuptake inhibitors (SSRIs). Three SSRIs (citalopram, escitalopram and sertraline) are known to be CYP2C19 substrates, and patients with very low CYP2C19 enzyme activity are at risk of developing long QT and ventricular arrhythmias when taking these SSRIs [48]. In this situation, some clinicians monitor patients on SSRIs using serial electrocardiograms (EKGs) rather than changing the prescription. The key to this approach is the availability of an endophenotype [49].

Conversely, patients at risk of developing coronary stent thrombosis on clopidogrel do not have an easily measured (noninvasive) endophenotype. In patients taking clopidogrel with CYP2C19 loss of function alleles, there are no simple affordable endophenotypes that can be similarly used for surveillance to monitor the risk of developing coronary stent thrombosis. Serial angiograms would be cost prohibitive and unnecessarily invasive, whereas serial platelet aggregometry has not proven to have sufficient predictive value to determine individual risk for stent thrombosis. Thus, without an endophenotype (such as QT interval for SSRIs), simply monitoring PCI patients with a CYP2C19 loss of function allele is undesirable. A change in prescription (either changing the drug or changing the dose) is indicated as outlined above.

\section{Pitfalls}

While the expansion of gene-based prescribing for antiplatelet agents now has significant momentum in routine practice, several challenges still exist. Rigorous longitudinal analyses are needed prospectively, to define the impact of this approach on medication adherence, quality of care, and cost in an overburdened healthcare infrastructure. Another key challenge related to gene-based drug prescribing has been the issue of pleiotropy, the principle that variation in one pharmacogene often impacts metabolism of multiple other classes of medications [50]. These potential obstacles are discussed below.

\section{Pleiotropy \& decision support}

CYP2C19 contributes to the metabolism of nearly $10 \%$ of all commonly prescribed drugs. As noted earlier, this enzyme oxidizes PPIs, TCAs and SSRIs. Genetic variability in CYP2C19 has therefore been associated with outcome in the context of many of these drugs [51]. CPIC has published a guideline addressing the relationship between CYP2C19 gene variants and some SSRIs [48]. Therefore, the clinical and scientific communities need to make sure that any implementation efforts deploying CYP2C19 genotyping to guide antiplatelet therapy will also provide decision support for patients using SSRIs metabolized by this enzyme. Further, the need for robust decision support (cross talk within electronic medical records that coordinates prescribing among multiple specialties) will almost certainly increase as more outcomes are linked to CYP2C19 gene variability for additional drug classes (e.g., PPIs). For example, it has been shown that CYP2C19 genotype predicts healing rates for Barrett's esophagus and eradication rates for Helicobacter pylori on triple therapy, in communities where the frequency of CYP2C19 gene variants is very high [52-54].

Another challenge that complicates implementation of decision support for pleiotropic pharmacogenes has been the issue of whether to deploy decision support for all patients that carry a loss of function allele (heterozygotes as well as homozygotes) or to intervene only with patients that have two loss of function alleles (homozygotes). At present, CPIC recommends acting on CYP2C19 genotype for heterozygotes and homozygotes considering clopidogrel, whereas CPIC recommends acting on CYP2C19 genotype for homozygotes only in the context of sertraline, citalopram or escitalopram [28,30]. Thus, the importance of robust decision support cannot be overstated. For direct to consumer genotyping, the lack of seamless integration into an electronic medical record may place patients at risk if decision support is not deployed within all clinical contexts where a pharmacogene variant has been proven to be actionable. The convergence of clinical informatics and laboratory genetics will therefore be key to success with CYP2C19 genotyping.

\section{Operational challenges}

Another potential obstacle has been turnaround time when genotyping is ordered reactively within the context of acute care. In this setting, the result may not be readily available by the time a PCI patient leaves the angiography 
suite. Even for institutions with an in-house genomics laboratory, turnaround time can be days to weeks. The time window can be even longer if results are sent to an outside lab. Rapid point-of-care genetic testing is being explored at some institutions where these devices appear to return results within 1-6 h [55]. Overall, however, the need to have pharmacogenetic data available in real time is leading to a shift from reactive testing (just in time) to pre-emptive testing (just in case). Pre-emptive genotyping may also lead to cost efficiencies. For example, if a provider orders CYP2C19 genotyping alone (without any other genetic tests), the out of pocket cost for the patient is likely to exceed $\$ 100$ (and sequencing the CYP2C19 gene to find rare variants may cost up to $\$ 1000$ ). The cost per gene, however, is driven down markedly if CYP2C19 genotype is obtained on a larger array containing multiple pharmacogenes. This is leading to an economy of scale, as providers shift to obtaining panels of genes in the context of primary care [56]. As this shift occurs, other potential challenges will develop as well. Simultaneous genotyping that generates decision support for many different classes of drugs holds the potential to overwhelm clinicians with electronic alerts. One proposed solution has been the integration of pharmacists and genetic counselors to deliver genetic information by using a team-based model [50].

\section{Future perspective}

CYP2C19 genotyping holds great promise to optimize outcome as clinicians select antiplatelet therapy for individual patients. Emerging data from randomized controlled trials support this claim, specifically for patients undergoing coronary intervention. A recent trial compared genotype-based treatment to usual care in 2488 patients undergoing PCI for an acute coronary syndrome (ACS) at ten European sites [57]. Patients in the usual care arm received prasugrel or ticagrelor. Patients in the genotype-based arm received clopidogrel unless they carried a loss-of-function CYP2C19 allele, in which case they were switched to prasugrel or ticagrelor. Within this framework, genotype-based treatment was noninferior to usual care with respect to reducing thrombotic events, and genotype-based treatment was associated with a lower incidence of bleeding [57].

Many questions remain, including when to choose prasugrel, and when to choose ticagrelor, as alternates to clopidogrel in patients with loss of function CYP2C19 alleles. The recent Intracoronary Stenting and Antithrombotic Regimen: Rapid Early Action for Coronary Treatment 5 (ISAR5-REACT) trial results demonstrated superiority of prasugrel compared with ticagrelor in ACS patients undergoing PCI [58]. Patients on prasugrel had fewer MACE events than patients on ticagrelor at 12 months (combined end point of death, nonfatal MI, or nonfatal stroke was $6.9 \%$ for prasugrel vs $9.3 \%$ for ticagrelor) [58]. Moreover, there was no increase in risk of bleeding with prasugrel compared with ticagrelor. Because prasugrel may be more affordable (due to loss of patent-protected exclusivity in 2017), a very compelling argument is emerging in favor of more wide use of this agent in the absence of specific contraindications (prasugrel is contraindicated in patients with active bleeding, a history of stroke, or age over 75 years).

Guidance is also needed for the use of CYP2C19 genotype in patients with other forms of vascular disease, beyond CAD. In patients using clopidogrel for peripheral arterial disease (PAD), CYP2C19 loss of function alleles are associated with diminished platelet response and a poorer prognosis after endovascular treatment [59]. In patients at risk for cerebrovascular disease, the Clopidogrel in High-Risk Patients with Acute Nondisabling Cerebrovascular Events (CHANCE) trial showed that the use of clopidogrel plus aspirin (vs aspirin alone) only reduces the frequency of stroke events in the subgroup of patients with normal CYP2C19 alleles [60]. Much additional work is therefore needed to identify the optimal approach to antiplatelet therapy in patients with transient ischemic attacks (TIA) and stroke.

As these questions are answered, and risk prediction algorithms become increasingly accurate, the convergence of pharmacogenetics and clinical informatics will guide us into the future. Large panels of pharmacogenes being tested pre-emptively in routine care must be linked to automated decision support within electronic medical records in a manner that is efficient and cost effective. The strong association between CYP2C19 gene variants and outcomes related to antiplatelet therapy is driving this field forward.

Financial \& competing interests disclosure

This work was funded in part by National Institutes of Health (1U01HG007253), and through a generous gift from T Denny Sanford creating the Imagenetics initiative (merging Internal Medicine and Genetics). The authors have no other relevant affiliations or financial involvement with any organization or entity with a financial interest in or financial conflict with the subject matter or materials discussed in the manuscript apart from those disclosed.

No writing assistance was utilized in the production of this manuscript. 


\section{Executive summary}

- In vivo, CYP2C19 bioactivates the antiplatelet drug clopidogrel.

- Loss of function variants in the CYP2C19 gene are associated with clopidogrel failure.

- Patients with a single loss of function CYP2C19 allele are at threefold increased risk for coronary stent thrombosis on clopidogrel when compared with patients with two normal CYP2C19 alleles.

- Risk for coronary stent thrombosis in patients with CYP2C19 loss of function alleles can be reduced by switching them from clopidogrel to alternate agents like prasugrel or ticagrelor.

\section{Open access}

This work is licensed under the Attribution-NonCommercial-NoDerivatives 4.0 Unported License. To view a copy of this license, visit http://creativecommons.org/licenses/by-nc-nd/4.0/

\section{References}

Papers of special note have been highlighted as: • of interest; $\bullet \bullet$ of considerable interest

1. Roth GA, Johnson C, Abajobir A et al. Global, Regional, and National Burden of Cardiovascular Diseases for 10 Causes, 1990 to 2015. J. Am. Coll. Cardiol. 70(1), 1-25 (2017).

2. Davi G, Patrono C. Platelet activation and atherothrombosis. N. Engl. J. Med. 357(24), 2482-2494 (2007).

3. Bowman L, Mafham M, Wallendszus $\mathrm{K}$ et al. Effects of aspirin for primary prevention in persons with diabetes mellitus. N. Engl. J. Med. 379(16), 1529-1539 (2018).

4. Gaziano JM, Brotons C, Coppolecchia R et al. Use of aspirin to reduce risk of initial vascular events in patients at moderate risk of cardiovascular disease (ARRIVE): a randomised, double-blind, placebo-controlled trial. Lancet 392(10152), 1036-1046 (2018).

5. Mcneil JJ, Wolfe R, Woods RL et al. Effect of aspirin on cardiovascular events and bleeding in the healthy elderly. N. Engl. J. Med. 379(16), 1509-1518 (2018).

6. Patrono C, Garcia Rodriguez LA, Landolfi R, Baigent C. Low-dose aspirin for the prevention of atherothrombosis. N. Engl. J. Med. 353(22), 2373-2383 (2005).

7. Berger JS, Brown DL, Becker RC. Low-dose aspirin in patients with stable cardiovascular disease: a meta-analysis. Am. J. Med. 121(1), 43-49 (2008).

8. Bertrand ME, Rupprecht HJ, Urban P, Gershlick AH. Double-blind study of the safety of clopidogrel with and without a loading dose in combination with aspirin compared with ticlopidine in combination with aspirin after coronary stenting: the Clopidogrel Aspirin Stent International Cooperative Study (CLASSICS). Circulation 102(6), 624-629 (2000).

9. Mehta SR, Yusuf S, Peters RJ et al. Effects of pretreatment with clopidogrel and aspirin followed by long-term therapy in patients undergoing percutaneous coronary intervention: the PCI-CURE study. Lancet 358(9281), 527-533 (2001).

10. Steinhubl SR, Berger PB, Mann JT 3rd et al. Early and sustained dual oral antiplatelet therapy following percutaneous coronary intervention: a randomized controlled trial. JAMA 288(19), 2411-2420 (2002).

11. Yusuf S, Zhao F, Mehta SR, Chrolavicius S, Tognoni G, Fox KK. Effects of clopidogrel in addition to aspirin in patients with acute coronary syndromes without ST-segment elevation. N. Engl. J. Med. 345(7), 494-502 (2001).

12. CAPRIE Steering Committee. A randomised, blinded, trial of Clopidogrel Versus Aspirin in Patients at Risk of Ischaemic Events (CAPRIE). Lancet 348(9038), 1329-1339 (1996).

13. Wiviott $\mathrm{SD}$, Braunwald $\mathrm{E}, \mathrm{McCabe} \mathrm{CH}$ et al. Intensive oral antiplatelet therapy for reduction of ischaemic events including stent thrombosis in patients with acute coronary syndromes treated with percutaneous coronary intervention and stenting in the TRITON-TIMI 38 trial: a subanalysis of a randomised trial. Lancet 371(9621), 1353-1363 (2008).

14. Wiviott SD, Braunwald E, McCabe $\mathrm{CH}$ et al. Prasugrel versus clopidogrel in patients with acute coronary syndromes. N. Engl. J. Med. 357(20), 2001-2015 (2007).

15. Wallentin L, Becker RC, Budaj A et al. Ticagrelor versus clopidogrel in patients with acute coronary syndromes. N. Engl. J. Med. 361(11), 1045-1057 (2009).

16. Amsterdam EA, Wenger NK, Brindis RG et al. 2014 AHA/ACC Guideline for the Management of Patients with Non-ST-Elevation Acute Coronary Syndromes: a report of the American College of Cardiology/American Heart Association Task Force on Practice Guidelines. J. Am. Coll. Cardiol. 64(24), e139-e228 (2014).

17. Neumann FJ, Sousa-Uva M, Ahlsson A et al. 2018 ESC/EACTS guidelines on myocardial revascularization. Eur. Heart J. 40(2), 87-165 (2019).

18. Antman EM, Hand M, Armstrong PW et al. 2007 focused update of the ACC/AHA 2004 guidelines for the management of patients with ST-elevation myocardial infarction: a report of the American College of Cardiology/American Heart Association Task Force on Practice Guidelines. J. Am. Coll. Cardiol. 51(2), 210-247 (2008). 
19. Mariani M, Mariani G, de Servi S. Efficacy and safety of prasugrel compared with clopidogrel in patients with acute coronary syndromes: results of TRITON-TIMI 38 trials. Expert Rev Cardiovasc Ther 7(1), 17-23 (2009).

20. Sangkuhl K, Klein TE, Altman RB. Clopidogrel pathway. Pharmacogenet Genomics 20(7), 463-465 (2010).

21. Hulot JS, Bura A, Villard E et al. Cytochrome P450 2C19 loss-of-function polymorphism is a major determinant of clopidogrel responsiveness in healthy subjects. Blood 108(7), 2244-2247 (2006).

22. Brandt JT, Close SL, Iturria SJ et al. Common polymorphisms of CYP2C19 and CYP2C9 affect the pharmacokinetic and pharmacodynamic response to clopidogrel but not prasugrel. J. Thromb. Haemost. 5(12), 2429-2436 (2007).

23. Umemura K, Furuta T, Kondo K. The common gene variants of CYP2C19 affect pharmacokinetics and pharmacodynamics in an active metabolite of clopidogrel in healthy subjects. J. Thromb. Haemost. 6(8), 1439-1441 (2008).

24. Mega JL, Close SL, Wiviott SD et al. Cytochrome p-450 polymorphisms and response to clopidogrel. N. Engl. J. Med. 360(4), 354-362 (2009).

25. Shuldiner AR, O'Connell JR, Bliden KP et al. Association of cytochrome P450 2 C19 genotype with the antiplatelet effect and clinical efficacy of clopidogrel therapy. JAMA 302(8), 849-857 (2009).

26. Scott SA, Sangkuhl K, Gardner EE et al. Clinical Pharmacogenetics Implementation Consortium guidelines for CYP2C19 genotype and clopidogrel therapy. Clin. Pharmacol. Ther. 90(2), 328-332 (2011).

- The original Clinical Pharmacogenetics Implementation Consortium (CPIC) guideline for clopidogrel.

27. Scott SA, Sangkuhl K, Stein CM et al. Clinical Pharmacogenetics Implementation Consortium guidelines for CYP2C19 genotype and clopidogrel therapy: 2013 update. Clin. Pharmacol. Ther. 94(3), 317-323 (2013).

28. Hicks JK, Sangkuhl K, Swen JJ et al. Clinical pharmacogenetics implementation consortium guideline (CPIC) for CYP2D6 and CYP2C19 genotypes and dosing of tricyclic antidepressants: 2016 update. Clin. Pharmacol. Ther. 102(1), 37-44 (2017).

29. Walton R, Kimber M, Rockett K et al. Haplotype block structure of the cytochrome P450 CYP2C gene cluster on chromosome 10. Nat. Genet. 37(9), 915-916 (2005).

30. de Morais SM, Wilkinson GR, Blaisdell J et al. The major genetic defect responsible for the polymorphism of $S$-mephenytoin metabolism in humans. J. Biol. Chem. 269(22), 15419-15422 (1994).

31. Gerbal-Chaloin S, Pascussi JM, Pichard-Garcia L et al. Induction of CYP2C genes in human hepatocytes in primary culture. Drug Metab. Dispos. 29(3), 242-251 (2001).

32. Peer CJ, Spencer SD, Vandenberg DA, Pacanowski MA, Horenstein RB, Figg WD. A sensitive and rapid ultra HPLC-MS/MS method for the simultaneous detection of clopidogrel and its derivatized active thiol metabolite in human plasma. J. Chromatogr. B Analyt. Technol. Biomed. Life Sci. 880(1), 132-139 (2012).

33. Kazui M, Nishiya Y, Ishizuka T et al. Identification of the human cytochrome P450 enzymes involved in the two oxidative steps in the bioactivation of clopidogrel to its pharmacologically active metabolite. Drug Metab. Dispos. 38(1), 92-99 (2010).

34. Wilke RA, Reif DM, Moore JH. Combinatorial pharmacogenetics. Nature Rev. Drug Discov. 4(11), 911-918 (2005).

35. Lewis JP, Backman JD, Reny JL et al. Pharmacogenomic polygenic response score predicts ischemic events and cardiovascular mortality in clopidogrel-treated patients. Eur Heart J. Cardiovasc. Pharmacother. 6(4), 203-210 (2019).

36. Relling MV, Klein TE. CPIC: Clinical Pharmacogenetics Implementation Consortium of the Pharmacogenomics Research Network. Clin. Pharmacol. Ther. 89(3), 464-467 (2011).

37. Whirl-Carrillo M, Mcdonagh EM, Hebert JM et al. Pharmacogenomics knowledge for personalized medicine. Clin. Pharmacol. Ther. 92(4), 414-417 (2012).

38. Wilke RA, Dolan ME. Genetics and variable drug response. JAMA 306(3), 306-307 (2011)

39. Shen DL, Wang B, Bai J et al. Clinical value of CYP2C19 genetic testing for guiding the antiplatelet therapy in a Chinese population. J. Cardiovasc. Pharmacol. 67(3), 232-236 (2016).

40. Cavallari LH, Lee CR, Beitelshees AL et al. Multisite investigation of outcomes with implementation of CYP2C19 genotype-guided antiplatelet therapy after percutaneous coronary intervention. JACC Cardiovasc Interv 11(2), 181-191 (2018).

-• IGNITE trial results demonstrate reduction of major adverse cardiovascular events (MACE) in loss of function (LOF) patients through changing the drug.

41. Weitzel KW, Elsey AR, Langaee TY et al. Clinical pharmacogenetics implementation: approaches, successes, and challenges. Am. J. Med. Genet. C Semin. Med. Genet. 166c(1), 56-67 (2014).

- First publication of decision support for this drug-gene relationship in a large commercial electronic medical record (EMR) EPIC.

42. Wiviott SD, Antman EM, Gibson CM et al. Evaluation of prasugrel compared with clopidogrel in patients with acute coronary syndromes: design and rationale for the trial to assess Improvement in Therapeutic Outcomes by Optimizing Platelet Inhibition with Prasugrel Thrombolysis In Myocardial Infarction 38 (TRITON-TIMI 38). Am. Heart J. 152(4), 627-635 (2006).

43. James SK, Roe MT, Cannon CP et al. Ticagrelor versus clopidogrel in patients with acute coronary syndromes intended for non-invasive management: substudy from prospective randomised Platelet Inhibition and Patient Outcomes (PLATO) trial. BMJ342, d3527 (2011). 
44. Hochholzer W, Ruff CT, Mesa RA et al. Variability of individual platelet reactivity over time in patients treated with clopidogrel: insights from the ELEVATE-TIMI 56 trial. J. Am. Coll. Cardiol. 64(4), 361-368 (2014).

45. Mega JL, Hochholzer W, Frelinger AL et al. Dosing clopidogrel based on CYP2C19 genotype and the effect on platelet reactivity in patients with stable cardiovascular disease. JAMA 306(20), 2221-2228 (2011).

46. Sherwood MW, Wiviott SD, Peng SA et al. Early clopidogrel versus prasugrel use among contemporary STEMI and NSTEMI patients in the US: insights from the National Cardiovascular Data Registry. J. Am. Heart Assoc. 3(2), e000849 (2014).

47. Bonaca MP, Bhatt DL, Oude Ophuis T et al. Long-term tolerability of ticagrelor for the secondary prevention of major adverse cardiovascular events: a secondary analysis of the PEGASUS-TIMI 54 trial. JAMA Cardiol. 1(4), 425-432 (2016).

48. Hicks JK, Bishop JR, Sangkuhl K et al. Clinical Pharmacogenetics Implementation Consortium (CPIC) guideline for CYP2D6 and CYP2C19 genotypes and dosing of selective serotonin reuptake inhibitors. Clin. Pharmacol. Ther. 98(2), 127-134 (2015).

49. Petry N, Lupu R, Gohar A et al. CYP2C19 genotype, physician prescribing pattern, and risk for long QT on serotonin selective reuptake inhibitors. Pharmacogenomics 20(5), 343-351 (2019).

50. Oberg V, Differding J, Fisher M, Hines L, Wilke RA. Navigating pleiotropy in precision medicine: pharmacogenes from trauma to behavioral health. Pharmacogenomics 17(5), 499-505 (2016).

51. Scott SA, Sangkuhl K, Shuldiner AR et al. PharmGKB summary: very important pharmacogene information for cytochrome P450, family 2, subfamily C, polypeptide 19. Pharmacogenet Genomics 22(2), 159-165 (2012).

52. Kawamura M, Ohara S, Koike T et al. The effects of lansoprazole on erosive reflux oesophagitis are influenced by CYP2C19 polymorphism. Aliment. Pharmacol. Ther. 17(7), 965-973 (2003).

53. Kawamura M, Ohara S, Koike T et al. Cytochrome P450 2C19 polymorphism influences the preventive effect of lansoprazole on the recurrence of erosive reflux esophagitis. J. Gastroenterol. Hepatol. 22(2), 222-226 (2007).

54. Furuta T, Sugimoto M, Shirai N, Ishizaki T. CYP2C19 pharmacogenomics associated with therapy of Helicobacter pylori infection and gastro-esophageal reflux diseases with a proton pump inhibitor. Pharmacogenomics 8(9), 1199-1210 (2007).

55. Wirth F, Zahra G, Xuereb RG, Barbara C, Fenech A, Azzopardi LM. Comparison of a rapid point-of-care and two laboratory-based CYP2C19*2 genotyping assays for personalisation of antiplatelet therapy. Int. J. Clin. Pharm. 38(2), 414-420 (2016).

56. Larson EA, Wilke RA. Integration of genomics in primary care. Am. J. Med. 128(11), 1251.e1251-1255 (2015).

57. Claassens DMF, Vos GJA, Bergmeijer TO et al. A genotype-guided strategy for oral P2Y12 inhibitors in primary PCI. N. Engl. J. Med. 381(17), 1621-1631 (2019).

58. Schupke S, Neumann FJ, Menichelli M et al. Ticagrelor or prasugrel in patients with acute coronary syndromes. N. Engl. J. Med. 381(16), 1524-1534 (2019).

- ISAR5-REACT trial showing superiority of prasugrel over ticagrelor in acute coronary syndrome/percutaneous coronary intervention (ACS/PCI) patients.

59. Guo B, Tan Q, Guo D, Shi Z, Zhang C, Guo W. Patients carrying CYP2C19 loss of function alleles have a reduced response to clopidogrel therapy and a greater risk of in-stent restenosis after endovascular treatment of lower extremity peripheral arterial disease. $J$. Vasc. Surg. 60(4), 993-1001 (2014).

60. Wang Y, Zhao X, Lin J et al. Association between CYP2C19 loss-of-function allele status and efficacy of clopidogrel for risk reduction among patients with minor stroke or transient ischemic attack. JAMA 316(1), 70-78 (2016).

- CHANCE trial showing that adding clopidogrel to aspirin reduces the risk of a new stroke only in patients with normal CYP2C19 alleles. 